\title{
EDITORIAL
}

\section{Clinical validation of precision medicine protocols: the last mile is the longest}

\author{
Michael P. Casaer ${ }^{1 *} \mathbb{D}$, Audrey De Jong ${ }^{2,3}$ and Anders Perner ${ }^{4}$
}

(c) 2020 Springer-Verlag GmbH Germany, part of Springer Nature

Advanced understanding of the complex pathophysiology of critical illness drives the development and optimization of sophisticated therapeutic protocols with the ambition to improve short- and long-term outcomes for every patient [1]. During the last decade, individualized, titrated, precision and/or target-driven approaches have proliferated [2]. Such strategies aim to replace or supplement more generic one-size-fits-all therapies or more simple clinical titration, through detailed measurements of markers of pathophysiological derangement followed by protocolized corrective or reactive interventions.

While the development of such more individualized treatment strategies can be challenging, their final clinical validation may prove to be the ultimate hurdle [3-5]. The replacement of nutritional targets based on simple bodyweight-age-gender-calculations with individual measurements of energy expenditure and nitrogen losses was a promising strategy according to observational data. Yet, this sophisticated and dynamic approach to ICUnutrition did not improve functional outcomes when evaluated against standard care in the EAT-ICU trial [6]. In severely burned patients, thermo-dilution-based hemodynamic monitoring created the opportunity to dynamically titrate fluid resuscitation based on patients' intrathoracic volumes and cardiac indices, rather than providing a fixed rate infusion calculated by body weight and total burned surface area [7]. Surprisingly, when tested in a randomized clinical trial (RCT) in 50 burned patients, the thermodilution-guided resuscitation increased the volume of crystalloids infused over the first

\footnotetext{
*Correspondence: michael.casaer@uz.kuleuven.ac.be

${ }^{1}$ Clinical Department and Laboratory of Intensive Care Medicine,

Department of Cellular and Molecular Medicine, KU Leuven, Herestraat 49, 3000 Leuven, Belgium

Full author information is available at the end of the article
}

$24 \mathrm{~h}$ by more than $10 \mathrm{~L}$ without improving cardiac output parameters or vital functions. The authors reported more pronounced subcutaneous edema in the thermodilutiongroup without further details [7].

Both these trials demonstrate the importance of evaluating novel therapies in randomized clinical trials before broad implementation of a new strategy, even when they appear intuitively and pathophysiologically superior.

From this perspective, it makes some sense that the "European guidelines on management of major bleeding and coagulopathy following trauma: 4th edition" formulated a recommendation graded $1 \mathrm{C}$ for the use of Viscoelastic Hemostatic Assay (VHA) guided correction of coagulopathy, while the more simple approach using Conventional Coagulation Tests (CCT) (prothrombin time, activated partial thromboplastin time, platelet counts and fibrinogen) received a $1 \mathrm{~A}$ recommendation [8]. VHA offers many potential advantages as compared to CCT (Fig. 1). This point-of-care technology reduces the turnaround time from blood sampling to the detection of coagulopathy [8]. Moreover, VHA provides functional analyses of clot formation (including the effect of anti-aggregation drugs), while CCT includes static parameters [8]. Importantly, the use of VHA-based strategies may reduce the consumption of blood products [8]. Yet, the expert-group writing the above guidelines did not find convincing evidence for improved patient outcomes using VHA-guided hemostatic therapy.

Baksaas-Aasen and co-investigators should be congratulated for the attempt to fill this evidence gap through the randomized ITACTIC trial of 390 trauma patients with major bleeding [9]. A major hemorrhage protocol (MHP), including early administration of tranexamic acid and transfusion of red cells, plasma and platelets in a 1-1-1-ratio was used in all patients as recommend [8]. Patients were randomized to the use of repeated VHA vs. CCT to augment the MHP [9].

\section{Springer}




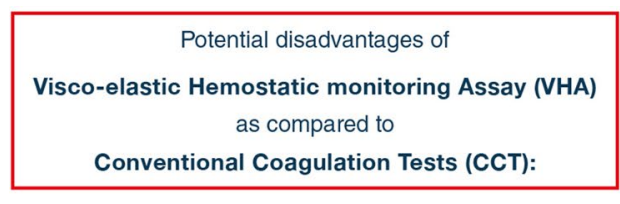

Increased complexity of care

Increased healthcare costs

Possibly, increased administration of hemostatic agents

Uncertain impact on patient-relevant outcomes

It induces seduction and "gizmo" idolatry of medical progress $(16,17)$

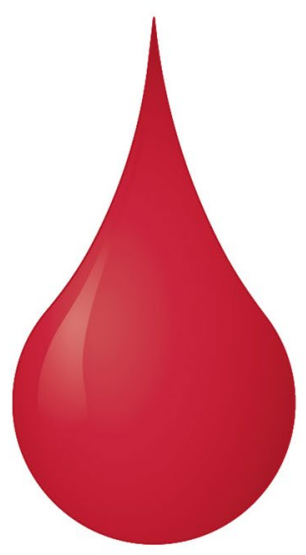

Visco-elastic Hemostatic monitoring Assay (VHA) as compared to

Conventional Coagulation Tests (CCT):

Reduced turnaround time from blood sampling to detection of coagulopathy

Potential reduction of blood product consumption

- Functional analyses of coagulation

- Evaluation of global clotting function, including the effect of anti-aggregation drugs

- Dynamical evaluation of clot formation and fibrinolysis

- Continuous display of kinetics for all clot formation stages (initiation, propagation, strength and dissolution)

Fig. 1 Proposed advantages and disadvantages of visco-elastic hemostatic assays

The methodology and statistical analysis appeared appropriate [10]. Indeed, the sample size was accurately calculated and the inclusion, exclusion criteria, main and secondary outcomes well-defined. Moreover, the randomization was well-conducted, the allocation list generated by an independent statistician, with block randomization by center, and block sizes unknown by the study team. The trial was pre-registered, with a prepublished protocol and a Data Monitoring Committee reviewed the outcome data.

However, the clinical team was unblinded, which could lead to differential treatment of the groups or the differential assessment of outcomes [11]. Another issue is the lower observed difference in effect size in the primary outcome between the two groups than considered for the sample size calculation. Moreover, despite the several subgroup analyses performed, randomization was not stratified for these and the analyses were not corrected for multiple testing.

The ITACTIC trial failed to demonstrate that VHAaugmented MHP improved mortality or reduced the use of massive transfusion in the first $24 \mathrm{~h}$. This happened despite reduced turn-around-time of the sample results (from $80 \mathrm{~min}$ [60-106] to $61 \mathrm{~min}$ [48-85]) and despite higher use of hemostatic products in the VHA-group [9]. In particularly more fibrinogen and platelets were used. The authors promote the results of patients with severe traumatic brain injury (TBI). In this subgroup, despite no difference in primary outcome, reduced 28-day mortality was observed. Subgroup analyses are common in RCTs, and particularly unreliable and affected by many factors [12]. Even if the subgroup analysis of patients with severe TBI was pre-defined, it comes with many limitations and it should be considered hypothesis-generating only as discussed by the authors.

Even if ITACTIC is a neutral RCT, its importance should not be underestimated [13]. If, based on the results, guideline committees dampen the recommendations for the use of VHA-augmented MHP, this may impact trauma care in several ways. First, care may be simplified so that staff with less VHA-experience should not be distracted from the basic diagnostic and therapeutic priorities because the VHA-generated figures and numbers may not dictate care $[8,14]$. This may also result in less use of hemostatic agents. At the organizational level, the need to implement VHA-augmented MHP should be reconsidered (until new evidence is generated). This will result in cost-savings as VHA requires hardware and reagents in emergency rooms, operating theaters and ICUs and specific training for on-site VHA-operators in these locations. These aspects will be undoubtedly addressed in the prospectively planned health economy analysis of ITACTIC.

We should, however be careful not to jump too fast to conclusions on de-adopting VHA, based on this "neutral" RCT [14]. Most of the participating centers didn't use VHA-augmented MHP in their clinical trauma routine prior to the RCT [9]. It thus remains possible that 
the impact of VHA in centers with longstanding VHAexpertise may be different. Also, centers operating in a different context with e.g. longer time from injury to admission and/or less stringent initial use of MHP might observe more frequent and severe coagulopathy [15]; such patients may benefit from VHA vs. CCT.

Above all, the ITACTIC trial should be an example for clinicians and researchers in all fields of critical care on how to validate a novel treatment modality.

\begin{abstract}
Author details
${ }^{1}$ Clinical Department and Laboratory of Intensive Care Medicine, Department of Cellular and Molecular Medicine, KU Leuven, Herestraat 49, 3000 Leuven, Belgium. ${ }^{2}$ University of Montpellier, INSERM U1046, CNRS UMR 9214, Montpellier, France. ${ }^{3}$ Anesthesia and Critical Care Department B, Saint Eloi Teaching Hospital, Centre Hospitalier Universitaire Montpellier, 34295 Montpellier Cedex 5, France. ${ }^{4}$ Department of Intensive Care, Rigshospitalet, University of Copenhagen, Copenhagen, Denmark.
\end{abstract}

\section{Funding}

MPC receives funding by Research Foundation Flanders (FWO) (Grant No. 1832817N), and Onderzoeksraad, KU Leuven (Grant No. C24/17/070).

\section{Compliance with ethical standards}

\section{Conflicts of interest}

MPC and ADJ state that they have no conflict of interest. AP receives grant support from the Novo Nordisk Foundation and Pfizer.

\section{Publisher's Note}

Springer Nature remains neutral with regard to jurisdictional claims in published maps and institutional affiliations.

Received: 7 October 2020 Accepted: 13 October 2020

Published online: 29 October 2020

\section{References}

1. Hawryluk GWJ, Aguilera S, Buki A et al (2019) A management algorithm for patients with intracranial pressure monitoring: the Seattle International Severe Traumatic Brain Injury Consensus Conference (SIBICC). Intensive Care Med 45:1783-1794
2. May F, Fourati S, Mekontso Dessap A, de Prost N (2020) Antiviral treatment strategy should be individualized in critically ill patients with severe influenza. Intensive Care Med 46:1292-1293

3. Helms J, Bion J, De Jong A (2019) Observational vs randomized: David vs Goliath for thromboprophylaxis in critically ill patients? Intensive Care Med 45:272-274

4. Moseby-Knappe M, Westhall E, Backman S et al (2020) Performance of a guideline-recommended algorithm for prognostication of poor neurological outcome after cardiac arrest. Intensive Care Med 46:1852-1862

5. Brohi K, Gruen RL, Holcomb JB (2019) Why are bleeding trauma patients still dying? Intensive Care Med 45:709-711

6. Allingstrup MJ, Kondrup J, Wiis J et al (2017) Early goal-directed nutrition versus standard of care in adult intensive care patients: the single-centre, randomised, outcome assessor-blinded EAT-ICU trial. Intensive Care Med 43(11):1637-1647

7. Holm C, Mayr M, Tegeler J et al (2004) A clinical randomized study on the effects of invasive monitoring on burn shock resuscitation. Burns 30:798-807

8. Rossaint R, Bouillon B, Cerny V et al (2016) The European guideline on management of major bleeding and coagulopathy following trauma: fourth edition. Crit Care 20:100

9. Baksaas-Aasen K (2020) Viscoelastic haemostatic assay augmented protocols for major trauma haemorrhage (ITACTIC): a randomized, controlled trial. Intensive Care Med. https://doi.org/10.1007/s00134-020-06266-1

10. Harhay MO, Casey JD, Clement M et al (2020) Contemporary strategies to improve clinical trial design for critical care research: insights from the First Critical Care Clinical Trialists Workshop. Intensive Care Med 46:930-942

11. Karanicolas PJ, Farrokhyar F, Bhandari M (2010) Practical tips for surgical research: blinding: who, what, when, why, how? Can J Surg J Canadien de Chirurgie 53:345-348

12. Brookes ST, Whitley E, Peters TJ, Mulheran PA, Egger M, Davey SG (2001) Subgroup analyses in randomised controlled trials: quantifying the risks of false-positives and false-negatives. Health Technol Assess (Winchester, England) 5:1-56

13. Leontiadis Gl (2016) How to interpret a negative study. Am J Gastroenterol 111:1506-1507

14. Young PJ, Nickson CP, Perner A (2020) When should clinicians act on nonstatistically significant results from clinical trials? JAMA 323:2256-2257

15. Khan S, Davenport R, Raza I et al (2015) Damage control resuscitation using blood component therapy in standard doses has a limited effect on coagulopathy during trauma hemorrhage. Intensive Care Med 41:239-247

16. Leff B, Finucane TE (2008) Gizmo idolatry. JAMA 299:1830-1832

17. Thimbleby H (2013) Technology and the future of healthcare. J Public Health Res 2:e28 